\title{
ELECTRONIC SPECTRA OF CATIONIC PAH AND PAH CLUSTERS
}

\author{
H. Friha ${ }^{1,2}$, G. Féraud ${ }^{1}$, T. Pino ${ }^{1}$, P. Parneix ${ }^{1}$, Z. Dhaouadi $^{2}$ \\ and $\mathrm{Ph}$. Bréchignac ${ }^{1}$
}

\begin{abstract}
An experiment designed for measuring the electronic spectra of PAH cations and PAH cluster cations has been set up, which is briefly described. The photodissociation spectra, reflecting the absorption spectra, of 2-methyl-naphthalene dimer cation have been recorded in the spectral ranges corresponding to the transitions from the ground state to both the excited charge resonance state and the first allowed local electronic excitation state.
\end{abstract}

\section{Introduction}

The presence of polycyclic aromatic hydrocarbons (PAHs) in the interstellar medium (ISM) was suggested in the mid-80s (Léger \& Puget 1984; Allamandola et al. 1985). Since then, their ubiquity and their important role in the physicochemical evolution of the ISM have been confirmed through many observational studies of the "Aromatic Infrared Bands" (AIBs; see the recent conference proceedings by Joblin \& Tielens 2011). Interstellar PAHs have also been proposed as possible carriers of some Diffuse Interstellar Bands (DIBs; Léger 1995). These absorption bands, seen in the spectra of reddened stars from the visible to the near infrared, constitute a major astrophysical issue. In recent years, high spatial resolution observations in the AIBs range have been conducted in specific regions like reflection nebulae. Some of them suggest the possible presence of PAH clusters in the transition region of the PhotoDissociation Regions (Rapacioli et al. 2005). Although this suggestion is still controversial such clusters can be considered as a model material to describe the particles intermediate in size between PAHs and very small grains (VSGs) (Berné et al. 2007). Since very little information is presently available on the physical and chemical properties of these clusters (Saigusa \& Lim 1995; Bréchignac et al. 2005), there is a need for new systematic experimental and theoretical studies.

\footnotetext{
1 Institut des Sciences Moléculaires d'Orsay (ISMO) CNRS Université Paris Sud, 91405 Orsay, France

${ }^{2}$ Laboratoire de Spectroscopie Atomique et Moléculaire, Faculté des Sciences, Tunis
} 
We report here some results of experiments which aim at gathering new laboratory data on the electronic spectra of cold gas phase PAHs, as well as those of their clusters, to be used for probing their participation to the interstellar extinction curve from the near infrared and visible (DIBs) to the UV (bump) spectral ranges. For this experimental goal PAHs cations and PAH cluster cations represent an excellent set of target species, since their spectra can be unambiguously assigned by using adequate combination of laser spectroscopy and mass spectrometry techniques. The molecule presently under investigation in this way is 2-methyl-naphthalene $\left(C_{11} H_{10}\right)$. The visible and near-UV absorption of its cation $C_{11} H_{10}^{+}$in rare gas matrices was early reported by Andrews et al. (1982): the first three transitions from the ground $D_{0}$ state to excited doublet states were found at $14903 \mathrm{~cm}^{-1}\left(D_{2}-D_{0}\right), 21137 \mathrm{~cm}^{-1}\left(D_{3}-D_{0}\right)$ and $25994 \mathrm{~cm}^{-1}\left(D_{4}-D_{0}\right)$ in Argon matrices. More specifically the results described in this paper focus on the electronic spectra of the gas phase dimer cation $\left(\mathrm{C}_{11} \mathrm{H}_{10}\right)_{2}^{+}$.

\section{Experimental}

A new way of forming $\mathrm{PAH}_{m}^{+}-\mathrm{Ar}_{n}$ cluster cations has been implemented in the experimental set-up "Icare" at ISMO (Orsay), giving us the capability to measure the electronic spectra of cold PAH cations in the gas phase through the "Ar-atom tagging" method (Pino et al. 1999; Bréchignac \& Pino 1999). In the just cited original set of measurements, the Van der Waals complex cations were selectively formed in their $v=0$ ground state, within the molecular beam while passing between the accelerating plates of the orthogonally-set Time-Of-Flight (TOF) spectrometer, thanks to resonantly enhanced two-photon laser ionization of jet-cooled corresponding neutrals. This method required careful wavelength adjustment of two different tunable dye lasers. In the present case, Van der Waals complexes of 2-methyl-naphthalene with Ar atoms are first formed in the expansion of a supersonic jet, while being irradiated - very close to the nozzle - by a pulsed laser beam (at a fixed wavelength in the 260 to $290 \mathrm{~nm}$ range) which photo-ionizes the molecules and clusters. The just formed cations are then injected (at least partly) through a skimmer and continue to flow with the molecular beam velocity. A second tunable pulsed (OPO) laser beam crossing downstream photo-dissociates the cations after some delay. As in the previous method the resulting bare $\mathrm{PAH}^{+}$ fragments are detected using a TOF mass spectrometer while scanning the laser wavelength from 470 to $690 \mathrm{~nm}$ (signal visible beam) and from $750 \mathrm{~nm}$ to $1400 \mathrm{~nm}$ (idler infrared beam). However in the present case the acceleration voltage needs to be pulsed and applied at the proper time corresponding to the transit time of the cations from the photoionization laser spot. The proper fine adjustment of the additional delay separating the photodissociation pulse from the TOF extraction pulse is a key factor for allowing unambiguous identification of the fragment ions peaks as well as their parent clusters ${ }^{3}$.

\footnotetext{
${ }^{3}$ This will be explained in more details in a forthcoming publication.
} 
If the jet expansion conditions are tuned to form PAH clusters, the same protocol allows to photodissociate a family of cluster cations of the type $P A H_{m}^{+}-A r_{n}$. After a careful identification of parent and daughter cations is made, the recording of a variety of spectra can be done. Interestingly as soon as a PAH dimer (or another multimer) is ionized the phenomena of charge resonance between the two moieties (or the adjacent units within the cluster) takes place, whose effect is to stabilize the ground electronic state of the cluster cation. Accordingly an upper-lying excited charge resonance state is created which can be reached from the ground state through a dipole-allowed optical transition. Both this transition (near $1100 \mathrm{~nm}$ ) and the first allowed one connecting the ground state to a locallyexcited state (near $570 \mathrm{~nm}$ ) have been recorded for the $\left(C_{11} H_{10}\right)_{2}^{+}$cationic dimer by using either the "idler" channel (near infrared) or the "signal" channel (visible) of the OPO laser. The obtained results are presented below.

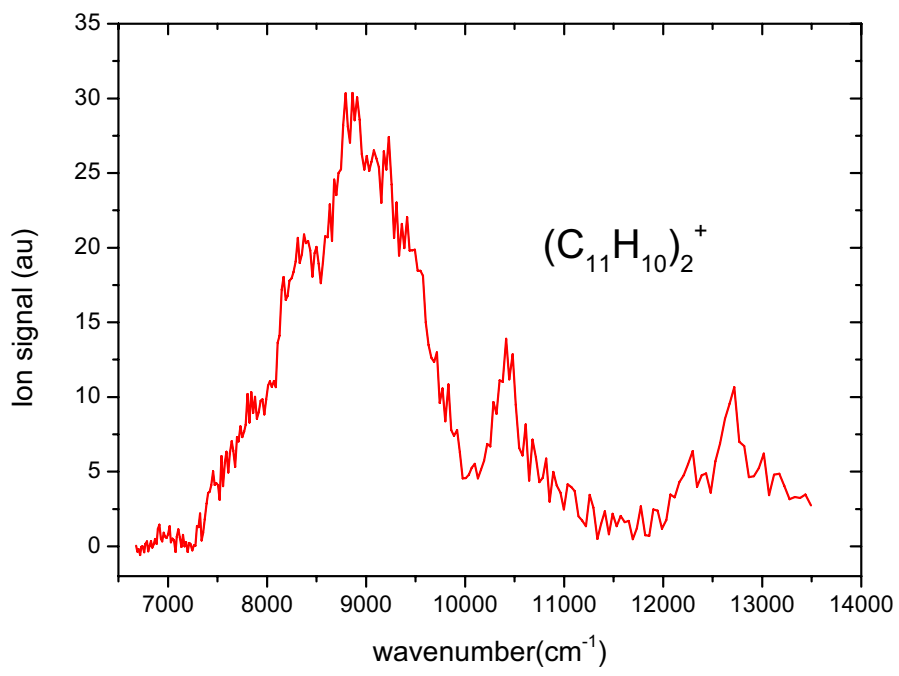

Fig. 1. Near-infrared photofragmentation spectrum of 2-methyl-naphthalene dimer cation. It was obtained by monitoring the signal generated out of $\left(\mathrm{C}_{11} \mathrm{H}_{10}\right)_{2}^{+}-\mathrm{Ar}$ in the mass peak assigned to $C_{11} H_{10}^{+}$fragment as a function of the OPO (idler) laser wavenumber.

\section{Results}

Figure 1 shows the photodissociation efficiency spectrum of the 2-methylnaphthalene dimer cation in the region of the transition from the ground (CR) to the excited $\left(\mathrm{CR}^{*}\right)$ charge resonance states. It exhibits a fairly broad main structure peaking near $8900 \mathrm{~cm}^{-1}$ (i.e. $1.10 \mathrm{eV}$ ) and extending from about 7200 to $13000 \mathrm{~cm}^{-1}$. A second, weaker, broad peak is seen near $12600 \mathrm{~cm}^{-1}$ (i.e. $1.56 \mathrm{eV})$. A peak of intermediate energy and intensity, possibly less broad than the other two, is seen near $10400 \mathrm{~cm}^{-1}$ (i.e. $1.29 \mathrm{eV}$ ). 
Figure 2 shows the photodissociation efficiency spectrum of the 2-methylnaphthalene dimer cations $\left(C_{11} H_{10}\right)_{2}^{+}-A r_{n}$ with $n=1$ and 2 in the visible region. It was found that the influence of binding a few more argon atoms to the PAH cluster remained barely detectable. We then concluded that it is negligible. It exhibits one main feature, peaking at $17427 \mathrm{~cm}^{-1}(2.16 \mathrm{eV})$ and degrading to the blue. It is assigned to the the origin band of the first allowed transition from the ground state to a locally-excited (LE) state, while the additional features could be due to associated vibronic bands. The bandwidth of the main feature is of the order of $500 \mathrm{~cm}^{-1}$, which is about half the value observed for the charge transfer transition. This transition should correspond to the $D_{2}-D_{0}$ first allowed electronic transition in the monomer (lying at $14903 \mathrm{~cm}^{-1}$ in Argon matrix (Andrews et al. 1982 ) and at $15120 \mathrm{~cm}^{-1}$, i.e. $1.87 \mathrm{eV}$, in the gas phase (Pino et al. to be published), which is blue-shifted by the stabilization of the ground state due to the charge resonance effect.

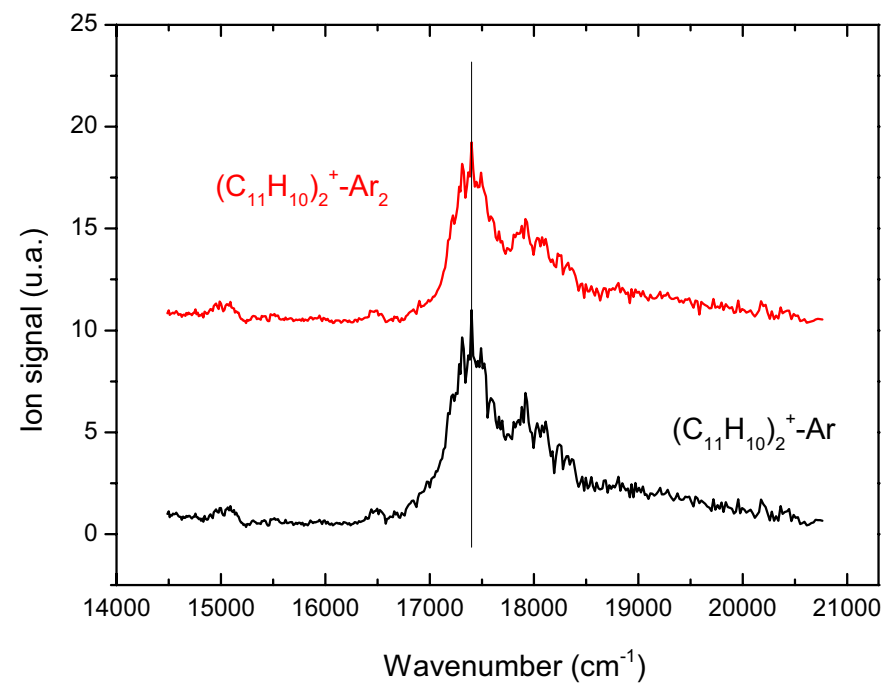

Fig. 2. Visible photofragmentation spectrum of 2-methyl-naphthalene dimer cation. It was obtained by monitoring the signal generated out of $\left(C_{11} H_{10}\right)_{2}^{+}-A r_{n}$ (for $\mathrm{n}=1$ and 2$)$ in the mass peak assigned to $C_{11} H_{10}^{+}$fragment as a function of the OPO (signal channel) laser wavenumber.

\section{Discussion and summary}

When a symmetric molecular homodimer M-M (where M stands for the name of the monomer molecule) is ionized, new basic quantum effects take place due to the equivalent character of the two configurations $\left(\mathrm{M}^{+}-\mathrm{M}\right.$ and $\left.\mathrm{M}-\mathrm{M}^{+}\right)$in which either one of the two moieties $M$ is carrying the charge (Bouvier et al. 2002). If $\mathrm{M}$ is an aromatic hydrocarbon and the monocation $M^{+}$is in its ground state, 
one electron has been ejected from the HOMO $\pi$ orbital. If $\mathrm{M}^{+}$is in one excited state, $\mathrm{M}^{+*}$, one electron from a lower $\pi$ orbital - lying below the HOMO - has been promoted to a higher-lying orbital. Thus, on the one hand, in the ground electronic state of the dimer cation, the two molecules play equivalent roles and a charge resonance (CR) effect takes place (Rapacioli et al. 2011). On the other hand, when an electronic transition is induced in the cationic dimer (of the type HOMO-n to HOMO in the present case), the two molecules are not equivalent and a locally-excited state is involved, in which the promoted orbital originates from the molecule which carries the charge (if not it would involve a promotion to a $\pi^{*}$ orbital, lying at a much higher energy).

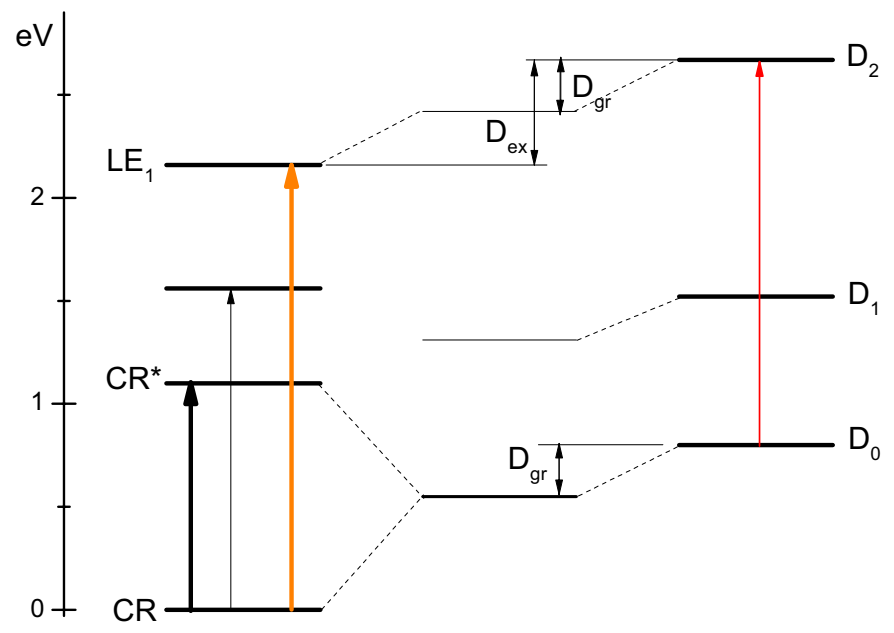

Fig. 3. Approximate energy levels diagram of the monomer and dimer cations of 2methyl-naphthalene, relevant for the present study.

The present new spectroscopic results on the electronic transitions in the 2methyl-naphthalene dimer cation, when put together with the spectroscopy of the monomer cation, allow to discuss the relative importance of the charge resonance effect, present in the ground state of the dimer cation, and the influence of the dimerization on the position of the excited state $\mathrm{M}^{+*}$. Indeed, assuming that the upper charge resonance state does not interact with any locally excited state, the charge resonance effect stabilization energy is equal to half the charge resonance transition energy of $1.10 \mathrm{eV}$, i.e. $C R=0.55 \mathrm{eV}$. This stabilization comes in addition to the contribution from the usual dispersion + induction interactions in clusters, which we call $D_{g r}$ (see Fig. 3). In the locally excited state these interaction forces also contribute to shift its position in energy relative to the $D_{2}$ excited state of the monomer. If we call this term $D_{e x}$ we have: $h \nu_{L E}=$ $h \nu_{D_{2}-D_{0}}+C R+D_{g r}-D_{e x}$. It follows that the change in the dispersion + induction energy is $\Delta D=D_{e x}-D_{g r}=0.26 \mathrm{eV}$. This relatively large value could indicate that the position in energy of the upper charge resonance state may be 
affected by an interaction with another nearby locally-excited state. A candidate for this is the state correlating with the $D_{1}$ excited state of the monomer.

On the other hand the origin of the weaker near-IR bands is not clear. Although the first one near $10400 \mathrm{~cm}^{-1}$ could be of vibronic nature (with $\approx 1500 \mathrm{~cm}^{-1}$ of excess vibrational energy), it seems difficult to be the case for the second one near $12600 \mathrm{~cm}^{-1}$, which then could be the above candidate, its relative weakness being in agreement with the symmetry-forbidden character of the $D_{1}-D_{0}$ transition, occuring at about $0.72 \mathrm{eV}$ in the naphthalene monomer cation, according to photoelectron data (Schmidt 1977; Maier et al. 2011). In conclusion, these experimental results are calling for a rather detailed theoretical study before they can be fully interpreted.

\section{References}

Allamandolla, L.J., Tielens, A.G.G.M., \& Barker, J.R., 1985, ApJ, 290, 25

Andrews, L., Kelsall, B.J., \& Blankenship, T.A., 1982, J. Phys. Chem., 86, 2916

Berné, O., Joblin, C., Deville, Y., et al., 2007, A\&A, 469, 575

Mayer, P.M., Blanchet, V., \& Joblin, C., 2011, J. Chem. Phys., 134, 244312

Bouvier, B., Brenner, V., Millié, P., \& Soudan, J.-M., 2002, J. Phys. Chem. A, 106, 10326

Bréchignac, Ph., Schmidt, M., Masson, A., et al., 2005, A\&A, 442, 239

Bréchignac, Ph., \& Pino, T., 1999, A\&A, 343, L49

Léger, A., 1995, in The Diffuse Interstellar Bands, edited by A.G.G.M. Tielens \& T.P. Snow (Kluwer Academic), 363

Léger, A., \& Puget, J.L., 1984, A\&A, 137, L5

Joblin, C., \& Tielens, A.G.G.M., 2011, PAHs and the Universe: A Symposium to Celebrate the 25th Anniversary of the PAH Hypothesis, EAS Publications Series, 46

Pino, T., et al., to be published

Pino, T., Boudin, N., \& Bréchignac, Ph., 1999, J. Chem. Phys., 111, 7337

Rapacioli, M., Joblin, C., \& Boissel, P., 2005, A\&A, 429, 193

Rapacioli, M., Spiegelman, F., Scemama, A., \& Mirtschink, A., 2011, J. Chem. Theory Comput., 7, 44

Saigusa, H., \& Lim, E.C., 1995, JACS, 117, 3862

Schmidt, W., 1977, J. Chem. Phys., 66, 828 